\title{
Ofidios y calor estivo: una enmienda necesaria en el texto de Plinio (Nat. XXIX 71)*
}

\author{
Arsenio Ferraces Rodríguez \\ Universidade da Coruña-Università degli Studi di Messina \\ a.ferraces@udc.es \\ ORCID iD: https://orcid.org/0000-0002-2103-036X
}

\section{Ophidians and summer heat: a necessary emendation in Pliny's text} (Nat. XXIX 71)

Según Plinio (Nat. XXIX 71) la culebra no es venenosa, pero puede llegar a serlo durante un período de tiempo por influjo de la luna (neque anguis uenenatus est nisi per mensem luna instigatus). La lectura per mensem carece de sentido porque los ofidios permanecen en letargo durante la época invernal. Por tanto, per mensem no puede hacer referencia a un mes cualquiera del año. Un pasaje de Gargilio Marcial y un testigo de la tradición indirecta de la Naturalis Historia apoyan la corrección per messem. De este modo, el pasaje en discusión se hace transparente y la doctrina de Plinio concuerda con la realidad: la culebra se vuelve venenosa en época estival, cuando tiene lugar la siega de la mies (messis).

Palabras clave: Plinio; Historia Natural; serpientes; tradición indirecta; enmienda del texto.
According to Pliny (Nat. XXIX 71), the snake is not a poisonous animal, but could become one during a specific period of time by influence of the moon (neque anguis uenenatus est nisi per mensem luna instigatus). The reading per mensem makes no sense because the ophidians remain in lethargy during winter. Therefore, per mensem cannot be referring to just any month of the year. A passage by Gargilius Martialis and a witness of the indirect tradition of the Naturalis Historia support the correction per messem. This way, the passage becomes transparent and Pliny's doctrine is consistent with reality: snakes become poisonous in summertime, at the time of harvest (messis).

Key words: Pliny; Natural History; snakes; indirect tradition; text emendation.

* Este artículo, que se inscribe en el marco del Proyecto FFI2013-45690-P (Ministerio de Ciencia, Innovación y Universidades), fue redactado durante una estadía como Visiting Professor en la Università degli Studi di Messina en los meses de octubre y noviembre de 2018. La consulta de los fondos bibliográficos de su nutrida biblioteca me permitió contrastar datos y dotar al trabajo del necesario aparato de referencias eruditas. Anna Maria Urso contribuyó a la aclaración de algunos puntos oscuros y libró al artículo de más de un error que al autor le había pasado desapercibido.

Copyright: (C) 2019 CSIC. Este es un artículo de acceso abierto distribuido bajo los términos de la licencia de uso y distribución Creative Commons Reconocimiento 4.0 Internacional (CC BY 4.0). 
Cómo citar este artículo / Citation: Ferraces Rodríguez, Arsenio 2019: «Ofidios y calor estivo: una enmienda necesaria en el texto de Plinio (Nat. XXIX 71)», Emerita 87 (2), pp. 305-316.

\section{Un pasaje oscuro en las ediciones de Plinio}

En un capítulo de la Naturalis Historia que expone los antídotos contra mordeduras de serpiente, todas las ediciones de la obra de Plinio dan el texto siguiente (Nat. XXIX 71):

Neque anguis uenenatus est nisi per mensem luna instigatus, et prodest uiuus conprehensus et in aqua contusus, si foueantur ita morsus.

El pasaje resulta oscuro debido a la ambigüedad de la expresión per mensem. La tradición manuscrita utilizada por los editores hasta la fecha sólo da variantes irrelevantes, si no banales: mensem $\mathrm{R}$, mense $\mathrm{VE}$, menses $\mathrm{dT}^{1}$. El contexto permite inferir que el autor especifica un período concreto durante el cual la culebra deviene venenosa ${ }^{2}$. Como unidad temporal de referencia adopta el mes, pero, en ausencia de mayor precisión, saber exactamente qué quiere decir nisi per mensem luna instigatus se antoja difícil.

Quienes han editado el libro XXIX de Plinio han hallado dificultad en el pasaje. Sin embargo, en lugar de admitir un locus desperatus y marcar el segmento en cuestión con las correspondientes cruces, han preferido dar el texto sin más, aun sin haberlo entendido. Mayhoff sugiere en el aparato crítico la posibilidad de que detrás de per mensem pudiese estar la lectura primo mense.

${ }^{1}$ Las siglas corresponden a los manuscritos siguientes: Florencia, Biblioteca Riccardiana, 488, s. IX ${ }^{\text {med. }}$ (= R); Leiden, Voss. Lat. F. 61, s. VIII-IX (= V); París, BNF, lat. 6795, s. IX ${ }^{2}$ (= E); París, BNF, lat. 6797, s. XII² (= d); Madrid, BN, 10042, s. XIII-XIV (= T). Para una lista que sobrepasa el centenar de manuscritos, con estudio de las relaciones entre ellos, reenvío al autorizado estudio de Reeve 2007.

${ }^{2}$ El término anguis suele ser una denominación genérica para las serpientes (cf. ThLL II, 51.76-84), aunque en ciertos contextos puede tener un sentido más restringido de serpiente de agua. Servio, ad Aen. 2, 204 afirma que las angues son animales de agua, las serpentes de tierra y los dracones de los templos, pero se trata más de una deducción contextual a partir de versos concretos de Virgilio que de una distinción basada en la realidad. El propio Servio admite que con frecuencia los nombres se confunden (sed haec significatio plerumque confunditur). Para más datos, v. Gaillard-Seux 2012, pp. 265-266. Útil relación de fuentes griegas y latinas sobre las serpientes en Lenz 1856, pp. 432-474. Para la terminología herpetológica ofrece un buen panorama Bodson 1986 (a propósito de anguis, p. 74). 
Aunque retiene también la lectura per mensem, Jones cree que la conjetura de Mayhoff podría ser justa y sugiere en nota a pie de página una explicación de naturaleza paleográfica. En cuanto a Ernout, se limita a dar el texto y las variantes, sin mencionar siquiera las dificultades advertidas por sus antecesores. Tampoco aluden al problema ni a posibles variantes textuales König y Hopp³.

Por su parte, quienes han intentado una traducción del pasaje, Jones y Ernout incluidos, han forzado sin excepción la frase problemática con el fin de darle un sentido que está todavía lejos de ser claro. Sirvan como muestra los ejemplos siguientes, que presento en orden cronológico de publicación: «La coleuvre n'est pas venimeuse, si ce n'est à certains jours du mois où elle est irritée par la lune» (Littré); «La coleuvre n'est pas venimeuse sauf pendant certaines périodes du mois où elle est excitée par la lune» (A. Ernout); «A snake too is venomous only when during the month it is angered by the moon» (W. H. S. Jones); «La biscia poi è velenosa solo per un certo periodo del mese, in cui è eccitata dalla luna» (U. Capitani); «Eine Schlange is nicht giftig, außer sie wird zu einer bestimmten Zeit im Monat durch die Wirkung des Mondes gereitzt ...» (R. König - J. Hopp); «La culebra no es venenosa, salvo unos días al mes por influjo de la luna» (E. Tarriño) ${ }^{4}$.

A juzgar por las propuestas anteriores, las traducciones del pasaje a lenguas modernas presentan, en general, un grado de oscuridad no menor que el de la propia frase latina. Aún más, a excepción de Jones, los traductores incurren en un inconfesado vicio de copia. Para sortear la dificultad, todos acuden a la versión del texto latino por Littré, ya sea mediante consulta directa o bien por vía indirecta, copiando unos de otros con pequeñas variantes, en un proceso de reutilización en cadena ${ }^{5}$. Los problemas del pasaje tampoco

${ }^{3}$ Ian y Mayhoff 1967, p. 393; Jones 1963, pp. 228-231; Ernout 1962, p. 44; König, Hopp 1991, pp. 227-228 y p. 284. Jones afirma en la nota citada (pp. 228-229) lo siguiente: «Mayhoff's primo mense would mean: "in the early part of the month." A contraction of primo might easily be taken for per, and the change of mense to mensem would naturally follow.»

${ }^{4}$ Las referencias de las ediciones y traducciones consultadas son, además de las citadas en la nota precedente, las que siguen: Littré 1850, p. 309; Capitani, Garofalo 1986, p. 325 (la traducción es de U. Capitani); König, Hopp 1991, p. 61; Cantó et alii 2002, p. 595 (la traducción es de E. Tarriño).

${ }^{5}$ No he tenido en cuenta la versión del pasaje por Lenz 1856, p. 459, que prescinde del conflictivo per mensem y lleva a cabo más una paráfrasis que una traducción propiamente dicha: «Es gibt auch eine Schlange, die nicht giftig is, wenn sie es nicht durch den Einfluss des Mondes wird.» 
escaparon a la observación del redactor de la voz 'mensis' en el ThLL, que incluye este ejemplo entre los testimonios del término dudosos ${ }^{6}$.

\section{Los reptiles en las fuentes antiguas: letargo invernal y agresividad canicular}

Tal como ha sido editada hasta la fecha, la frase de Plinio es dificultosa, básicamente porque el giro per mensem, en singular, no delimita por sí solo un período específico y requeriría algún elemento adicional que el contexto no permite suplir. El naturalista resulta menos impreciso en otros lugares en donde emplea la misma expresión. Merecen ser citados al respecto, por su eficacia en términos de contraste, Nat. X 211, Homo genitus premitur somno per aliquot menses, dein longior in dies uigilia; y Nat. XVIII 291, ... ac, ne per omnes menses timeretur (= iniuria), earum quoque lege diuisum ... En ambos casos Plinio utiliza el término en plural, menses, pero añade un adjetivo, indefinido y distributivo, respectivamente, que facilita la comprensión del dato temporal. Frente al desnudo per mensem de Nat. XXIX 71 cabe citar igualmente un pasaje en el que Columela indica cuál es el momento propicio para hacer la siembra en zonas templadas?: XI 2.20, Trimestrium quoque satio non est aliena huic tempori, quamuis tepidis regionibus melius administretur per mensem Ianuarium. Con su concreción cronológica, la expresión per mensem Ianuarium, empleada por Columela, pone de manifiesto el carácter absolutamente inconcreto del per mensem pliniano.

En general, editores y traductores de Plinio no han prestado atención a un hecho real: al igual que otros reptiles, durante el período invernal la culebra permanece en letargo y es inofensiva. Por contra, su actividad y su agresividad máximas coinciden con la época estiva ${ }^{8}$. Además de mencionar la naturaleza fría de las serpientes, convertida incluso en motivo literario, las fuentes antiguas describen con precisión ese doble período en los ofidios, el de letar-

6 ThLL VIII 746.79.

${ }^{7}$ Utilizo la edición de Rodgers 2010.

${ }^{8}$ Datos abundantes sobre la creencia en la agresividad de las serpientes en época estival los ofrece un documentado trabajo de Trinquier 2012, particularmente, pp. 182-191. Entre las fuentes antiguas son reseñables, entre otras, Nic., Th. 121-123, 469-476; Cels. V 27.10; Verg., Georg. III 432-434. Véase, al respecto, el comentario de los citados pasajes de Nicandro en Jacques 2002, pp. 91-92 y pp. 141-143, respectivamente. 
go y el de actividad, respectivamente 9 . El caso más notorio es el de Galeno, quien refiere que durante los meses fríos las serpientes se esconden y no comen, y que su veneno tiene tan poco poder que pueden ser manejadas sin peligro $^{10}$. En cambio, según el propio Galeno, en la canícula el calor las abrasa hasta tal punto que no encuentran descanso ${ }^{11}$. Por su carácter explícito resulta oportuno citar por extenso uno de los pasajes concernidos, Loc. aff. II 5 (VIII 132-133 Kühn), acompañado de su traducción ${ }^{12}$ :

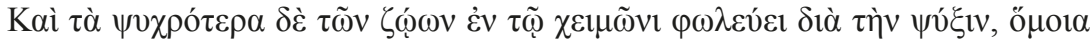

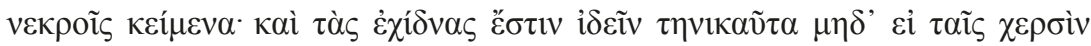

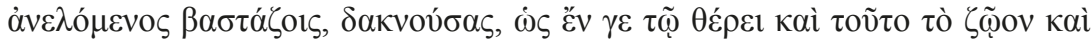

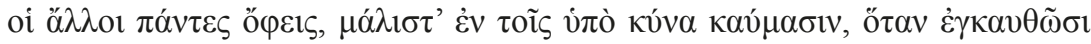

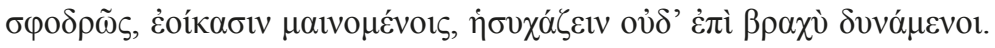

Durante el invierno aquellos animales que son de constitución especialmente fría se aletargan a causa del frío y permanecen como muertos. Se puede ver que en esta estación las víboras no muerden ni siquiera cuando se las coge con las manos. En cambio, en verano, y particularmente bajo el rigor de la canícula, cuando el calor los abrasa intensamente, tanto este bicho como los demás ofidios parecen presa de la locura y no pueden encontrar ni un instante de descanso.

Aunque de modo más sucinto, otras fuentes inciden en detalles similares. Un hecho en particular es mencionado de modo recurrente, el de que los reptiles se muestran agresivos en el período estivo, especialmente durante la canícula. Llevando al extremo la asociación entre calor y actividad de los ofidios, Nicandro refiere incluso que éstos son especialmente peligrosos durante las horas centrales del día, «cuando los pastores buscan el fresco, tras abandonar sus

9 Ejemplos de empleo de la naturaleza fría de las serpientes como motivo literario se encuentran en Theoc. XV 58; Verg., Ecl. III 93, VIII 72; Mart. VII 87.7, XII 28.5; Lucan. VI 488-489.

${ }^{10}$ En el mismo sentido Thphr., Fr. 10 Jacques (=Fr. 11 Rose); Plin., Nat. VIII 139.

${ }^{11}$ Pasajes notables al respecto son también Nic., Th. 469-476; Cels. V 27.10; Verg., Georg. III 432-434.

${ }_{12} \mathrm{Al}$ margen de las citadas más arriba a propósito de la frase pliniana en discusión, tanto la traducción del presente pasaje como todas las demás de este artículo son de mi entera responsabilidad. 
tareas $\gg{ }^{13}$. Por su parte, el propio Plinio (Nat. XXXII 55) atribuye a la autoridad de un tal Trásilo la doctrina de que el calor atormenta a las serpientes cuando el Sol está en el signo de Cáncer, es decir, en cómputo actual de calendario, del 21 de junio al 22 de julio ${ }^{14}$. De la información explícita de las fuentes antiguas, así como del hecho empírico de la permanencia de las serpientes en estado vegetativo en época invernal, se desprende una conclusión palmaria: la de que en el pasaje de Plinio per mensem no puede hacer referencia a un mes cualquiera del año. Y ahí radica el problema de las traducciones arriba referidas.

\section{Un pasaje de Gargilio Marcial}

Es Gargilio Marcial, autor tardoantiguo al que la tradición manuscrita atribuye unas Medicinae ex oleribus et pomis, quien aporta un dato precioso para sanar e interpretar el paso pliniano ${ }^{15}$. Entre las propiedades del serpol (serpillum) cita Gargilio su eficacia como repelente contra las serpientes. En ese contexto afirma que los segadores (messores) lo añadían a la comida para evitar ser mordidos en caso de apoderarse de ellos el sueño (Garg. Mart., Med. 39) ${ }^{16}$ :

Serpentes et omnia animalia uenenata adusti (= serpylli) nidor repellit. Ideo et messoribus in cibo miscetur ut, si fatigatos forte somnus oppreserit, tuto quiescant contra animalia quae id temporis uenenatis morsibus saeuire consuerunt.

La exhalación que desprende el serpol al arder repele las serpientes así como cualquier otra alimaña venenosa. Esa es la razón de que a los segadores se lo

${ }^{13}$ Nic., Th. 469-473; Plinio (Nat. XI 87-88) afirma lo mismo del escorpión, citando como fuente a Apolodoro: Apollodorus auctor est ... uenenum omnibus medio die, cum incanduere solis ardoribus, itemque, cum sitiunt, inexplebiles potus.

${ }^{14}$ Plin., Nat. XXXII 55, Thrasyllus auctor est ... cum sol sit in cancro, serpentes torqueri.

${ }^{15}$ La edición crítica de referencia es la de Maire 2002. Sobre el autor y su obra, además de la edición citada, presentan todavía interés las síntesis de Stadler 1910, Keil 1999, Fischer 2000.

${ }^{16}$ También Plin., Nat. XX 245 señala como repelente eficaz contra las serpientes el procedimiento de quemar serpol, así como la conveniencia de tomarlo en poción contra las mordeduras venenosas. Con menor concreción, Dioscórides (III 38) advierte que el serpol es eficaz contra las serpientes tanto bebido como aplicado en cataplasma. Para la descripción y las propiedades de la planta desde un punto de vista actual remito a Font Quer 2003, pp. 700-702. Nicandro (Th. 21-25) y Virgilio (Georg. III 435-436) advierten igualmente del peligro de dormir al aire libre en época estival. Remito, para otros detalles, a Jacques 2002, pp. LXXXIV-LXXXVI. 
mezclen también en la comida, para que, en la eventualidad de que rendidos de fatiga los venza el sueño, puedan descansar seguros y a salvo de las alimañas que en esa época suelen atacar con mordedura venenosa.

La mención de los segadores por Gargilio aporta información clave en relación con el pasaje de Plinio. La siega del cereal (messis) tiene lugar en época estival, hasta el punto de que el mismo término designaba por metonimia el calor tórrido de la canícula y la canícula misma ${ }^{17}$. Dos elementos fundamentales en Gargilio -la actividad de la serpiente y el momento preciso del año- están presentes también en Plinio, con sólo corregir per mensem en per messem en la frase en discusión. Si bien con empleo del plural en lugar del singular, el propio Plinio recurre otras veces a la misma expresión, como cuando indica que plantas como la tapsia (Nat. XIII 125), la adormidera (Nat. XX 61) o el moral (Nat. XXIII 140) han de ser recolectadas durante el estío (per messes) con el fin de extraer su jugo para uso médico ${ }^{18}$. De modo que, además de la información derivada de Gargilio Marcial, la crítica interna conduce también hacia una enmienda per messem en Nat. XXIX 71.

\section{Un testigo de tradición indirecta de la obra de Plinio}

Un testimonio de tradición indirecta incide también en la necesidad de corregir el texto de Plinio en este punto. Se trata de las Curae quae ex hominibus atque animalibus fiunt $(=$ Cur. anim.), un recetario médico resultante de una reelaboración de los libros 28-30 de la Naturalis Historia. Su época de redacción debe de situarse hacia el siglo $\mathrm{V}$, a juzgar por algunas coincidencias con el De medicamentis de Marcelo Empírico ${ }^{19}$. El capítulo 62, Curae quae ex angue fiunt, consta de veintitrés recetas, basadas en otros tantos pasajes en que Plinio hacía referencia a la culebra. La primera receta tiene como fuente precisamente el pasaje pliniano en discusión:

${ }^{17} O L D$, s. u. 'messis'; ThLL VIII 857.35-45.

${ }_{18}$ Plin., Nat. XIII 125, quidam caulem incidunt per messes et in ipsa excauant radice, quo sucus confluat; XX 61, carpitur (= papauer) per messes inciso caule, conditur fictili nouo, ad multa praeclarus; XXIII 140, Radix (= mori) per messes incisa sucum dat aptissimum dentium dolori.

${ }^{19}$ Edición en Ferraces Rodríguez 2015. Para la colocación de la obra en época próxima a Marcelo, cf. pp. 68-77. 


\begin{tabular}{|c|l|}
\hline \multicolumn{1}{|c|}{ Plin., Nat. XXIX 71-72 } & \multicolumn{1}{c|}{ Cur. anim. 62.1 } \\
\hline $\begin{array}{l}\text { Neque anguis uenenatus est nisi per } \\
\text { mensem luna instigatus, et prodest uiuus } \\
\text { conprehensus et in aqua contusus, si fouean- } \\
\text { tur ita morsus. }\end{array}$ & $\begin{array}{l}\text { Saeuitiam maiorem habent (= angues) } \\
\text { circa tempus messis nona luna surgente. } \\
\text { Prodest tamen ut uiui capiantur et in aqua } \\
\text { conterantur et exinde uulnera illorum qui ab } \\
\text { angue uel serpente percussi sunt liniantur. }\end{array}$ \\
$\begin{array}{l}\text { La culebra no es venenosa a no ser } \\
\text { [??] estimulada por la luna; y es eficaz } \\
\text { apresarla viva, majarla en agua y aplicar un } \\
\text { fomento en la mordedura. }\end{array}$ & $\begin{array}{l}\text { La culebra muestra su agresividad } \\
\text { máxima hacia el momento de la siega, } \\
\text { cuando aparece la novena luna. Es eficaz } \\
\text { apresarla viva, majarla en agua y extender } \\
\text { el líquido sobre la herida de quien ha sido } \\
\text { mordido por culebra o por serpiente. }\end{array}$ \\
\hline
\end{tabular}

En coincidencia con Gargilio, que asocia época de siega y agresividad del ofidio (messores, saeuire), el autor de las Curae afirma también que esa agresividad (saeuitia) es mayor hacia el momento de la siega. La expresión empleada, circa tempus messis, apenas permite dudar acerca de la lectura que daba el manuscrito de Plinio utilizado por el autor de esta reescritura: per messem, y no per mensem, era la forma empleada por ese testigo de la tradición directa de la Naturalis Historia hoy perdido, pero que tuvo existencia en una fecha tan temprana como el siglo $\mathrm{V}^{20}$. La aportación de las Curae en relación con Plin., Nat. XXIX 71 no reside en su doctrina, que, por lo demás, es conocida a través de otras fuentes, sino en que, en cuanto testimonio privilegiado de la tradición indirecta, viene a corroborar de modo definitivo la enmienda per messem en un pasaje que hasta ahora se resistía a nuestra comprensión.

${ }^{20}$ Aunque carece de incidencia real sobre la enmienda del pasaje de Plinio, que se trata del tiempo de la siega se desprende también de la precisión nona luna surgente. El término luna hace referencia aquí al ciclo lunar completo (cf. ThLL VII 1833.25-51), con sus cuatro fases, luna nueva, luna llena, cuarto creciente y cuarto menguante. El participio surgente es una referencia palmaria a la luna nueva. En la agricultura de zonas templadas todavía hoy la siega del cereal tiene lugar hacia el mes de agosto. Dado que el calendario lunar y el calendario solar no coinciden, el inicio de la 'novena luna', es decir la luna nueva del noveno ciclo lunar, puede acaecer hacia finales del mes de agosto o en los primeros días de septiembre. Quizás de ahí la cautela del autor de las Curae al utilizar una expresión aproximativa mediante el empleo de la preposición circa. Pero es messis el término que tiene su origen en el pasaje de Plinio y, por tanto, el dato relevante para la enmienda de éste. 


\section{Una deturpación sencilla: mensem es una hipercorrección de copista}

Confirmada la lección per messem tanto por crítica interna como por cotejo con otras fuentes, el proceso deturpatorio deviene transparente. La forma mensem es una hipercorrección de copista en un momento de la transmisión temprano, común en cualquier caso a los manuscritos utilizados por los editores hasta la fecha. El error, banal en términos de historia de la lengua, se ha visto favorecido por la concurrencia de dos hechos evolutivos: la simplificación de consonantes geminadas y el enmudecimiento de $n$ ante $s$ desde la época más remota de la lengua latina. La simplificación de las geminadas dio lugar al empleo indistinto de grafía simple o de geminada para consonantes realizadas ya comúnmente como simples ${ }^{21}$. La caída de $n$ ante $s$, por su parte, está documentada en latín desde los más tempranos testimonios epigráficos. Pero mientras en la época clásica el conservadurismo de la lengua escrita hizo perdurar, como norma, el grupo $n s$, en época tardía y altomedieval se produjo un elevado grado de confusión, con la frecuente caída de $n$, pero también con su restitución artificiosa en palabras que carecían de la misma ${ }^{22}$. La documentación acerca de tales cambios es abundante. Tratando de regularizar la grafía y adaptarla a la del latín clásico, la Appendix Probi postula a veces la recuperación de la $n$, como en la entrada 76 (ansa non asa) y en la 152 (tensa non tesa) ${ }^{23}$. En cambio, en otros casos, atendiendo al carácter advenedizo de tal consonante, defiende su supresión, como acontece en las entradas 19 (Hercules non Herculens), 75 (formosus non formunsus) o 123 (occasio non occansio). Una hipercorrección similar está detrás de grafías como thensaurus, quadragensimus o profensore, entre otras atestiguadas con

${ }^{21}$ A propósito de la simplificación de geminadas me permito remitir aquí únicamente a la reciente síntesis de Stotz 1996, pp. 312-314.

${ }^{22}$ Además de las grafías $\sin N$ en los epitafios de los Escipión, mencionan la caída de $n$ ante $s$ Varro, Ling. V 108; Quint., Inst. I 7.29, consules exempta littera $\mathrm{n}$ legimus; Vel., Gramm. VII 79. Este último afirma, además, que Cicerón pronunciaba foresia, Megalesia, Hortesia en lugar de forensia, Megalensia, Hortensia. Para otros ejemplos Stotz 1996, pp. 294-297.

${ }^{23}$ La edición de referencia es todavía hoy la de Baehrens 1922 (repr., Groningen, 1967). Útil bibliografía comentada al respecto en Quirk 2005. Nueva transcripción del texto a partir del manuscrito, con la finalidad de poner de relieve los problemas de la edición de Baehrens, en Powell 2007. En el caso de la entrada 152, Powell (p. 699) duda acerca de la lectura real del manuscrito, que puede estar referida a los términos tensa o mensa. 
frecuencia en las fuentes ${ }^{24}$. Ese doble fenómeno de la caída de $n$ ante $s$ y el de su restablecimiento inmotivado, en concurrencia con la simplificación de consonantes geminadas, daba lugar a situaciones ambiguas que, en el ámbito específico de la transmisión de los textos, cada copista resolvía de manera particular. Dado que, a una sola pronunciación, $-s-$, podían corresponder tres grafías distintas, $-s-$, $-s s-$ y $-n s-$, éstas eran susceptibles de ser utilizadas indistintamente por los copistas, según la intensidad de su formación o su grado de atención en el momento de copia. En nuestro caso, en la práctica las formas mesem, messem y mensem eran pronunciadas del mismo modo y podían corresponder indistintamente al acusativo de mensis o al de messis ${ }^{25}$. $\mathrm{Y}$ ese debe de haber sido el origen de la grafía per mensem, con empleo de $s$ simple y adición de una $n$ espuria, en Plin., Nat. XXIX 71. De acuerdo con los datos extraídos del aparato crítico de las ediciones de Plinio, para el término messis, 'siega, cosecha, estío', además de la forma mensis aquí enmendada, en la obra del naturalista está documentada la propia grafía clásica, messis, y la variante sin geminada, mesis. A su vez, el término mensis, 'mes', se encuentra también atestiguado con las grafías mesis, messis y menssis ${ }^{26}$.

\section{Todavía una precisión final}

En el panorama de la transmisión de los textos antiguos en época altomedieval tanto el caso aquí estudiado como la obra a la que pertenece no constituyen excepción. La misma confusión está atestiguada en la tradición manuscrita de otros autores. Como ejemplos conspicuos caben ser citados pasajes de Manilio (III 598), de Nemesiano (Ecl. IV 36) o de Ausonio (251.3). Merece ser traído a colación también el caso de Columela, por cuanto tres manuscritos, que llevan las siglas $\mathrm{S}, \mathrm{A}$ y $\mathrm{R}$ en la edición más reciente, dan en varios lugares la grafía mensem en lugar de messem $\mathrm{y}$, a la inversa, messem en lugar de mensem $^{27}$.

\footnotetext{
${ }^{24}$ Para otros ejemplos, con datos sobre fuentes, Stotz 1996, p. 296.

${ }^{25}$ A las tres posibles grafías del término mensis hace referencia explícita Stotz 1996, pp. 294-295: «Bei mensis setzen sich die Schreibungen mit mes- und mess- (vgl. it. mese u. a. m.) im MA fort ...».

${ }^{26}$ Véase, al respecto, ThLL VIII 745.75-78.

27 A propósito del pasaje de Manilio, remito a ThLL VIII 746.78-79; para los de Nemesiano y Ausonio, VIII, 857, 43-45. La lección mensem en lugar de messem es la que dan los
} 
Con todo, en estricto rigor, antes que de deturpación o de faltas de copista, en casos como los citados sería más justo hablar de polimorfismo gráfico durante el acto de copia. Tratándose de textos antiguos, cuya única forma de transmisión es a través de la escritura, es verosímil que dificultades como la originada por el acusativo mensem en lugar de messem sean tales sólo para nosotros, atentos como estamos a la forma escrita antes que a la realización oral de una palabra. Pero dichas dificultades podrían haber sido menores, o incluso inexistentes, en el caso de los copistas altomedievales y sus coetáneos, que tendrían presente la pronunciación real del término. Quizás para ellos la grafía del mismo, en cualquiera de sus variantes, tuvo una incidencia menor en cuanto a la comprensión de su sentido. Al margen de esta precisión, que atañe a la transmisión del texto, pero no a su redacción primera, la corrección de la grafía mensem en messem en Plin., Nat. XXIX 71 es obligada y necesaria, dada la época en que escribe el autor. El texto debe ser restablecido como sigue: Neque anguis uenenatus est nisi per messem luna instigatus ..., «La culebra no es venenosa a no ser por la época de la siega, cuando es estimulada por la luna ...».

\section{BIBLIOGRAFÍA}

Baehrens, W. 1922: Sprachlicher Kommentar zur vulgärlateinischen Appendix Probi, Halle (Saale) (repr., Groninga, 1967).

Bodson, L. 1986: «Observations sur le vocabulaire de la zoologie antique: les noms de serpents en grec et en latin», Documents pour l'histoire du vocabulaire scientifique 8, pp. 65-119.

Cantó, J. et alii 2002: Plinio. Historia Natural. Madrid.

Capitani, U. y Garofalo, I. 1986: Gaio Plinio Secondo. Storia Naturale. IV. Medicina e farmacologia. Libri 28-32, Turín.

Ernout, A. 1962: Pline l'Ancien. Histoire Naturelle. Livre XXIX, París.

Ferraces Rodríguez, A. 2015: Curae quae ex hominibus atque animalibus fiunt. I. Introducción y edición crítica, Santiago de Compostela.

manuscritos SAR de Columela en VII 3.11 (Rodgers, p. 290) y en VIII 10.20 (Rodgers, p. 343). La grafía messem en lugar de mensem está atestiguada en el anónimo Liber de arboribus 25 (Rodgers, p. 579). Para otros ejemplos de mensis en lugar de messis, v. Stotz 1996, p. 296, nota 204. 
Fischer, K. D. 2000: «Q. Gargilius Martialis», en R. Herzog y P. L. Schmidt (eds.), Nouvelle histoire de la littérature latine 4, Turnhout, pp. 307-311.

Font Quer, P. 2003: Plantas medicinales. El Dioscórides renovado, Barcelona.

Gaillard-Seux, P. 2012: «Le serpent, source de santé: le corps des serpents dans la thérapeutique gréco-romaine», Anthropozoologica 47, pp. 263-289.

Ian, L. y Mayhoff, C. 1967: C. Plinius Secundus. Naturalis Historia, vol. IV, Stuttgart.

Jacques, J. M. 2002: Nicandre. Oeuvres, II. Les thériaques. Fragments iologiques antérieurs à Nicandre, París.

Jones, W. H. S. 1963: Pliny. Natural History, Vol. VIII. Libri XXVIII-XXXII, Cambridge, MA.

Keil, G. 1999: «Gargilius Martialis», en LexMA IV, Stuttgart, col. 115-116.

König, R. y Hopp, J. 1991: C. Plinius Secundus d. Ä. Naturkunde. Lateinischdeutsch. Bücher XXIX/XXX, Múnich-Zúrich.

Lenz, H. O. 1856: Zoologie der alten Griechen und Römer, Wiesbaden.

Littré, M. É. 1850: Histoire Naturelle de Pline, avec la traduction en français, Tome second. París.

Maire, B. 2002: Gargilius Martialis. Les remèdes tirés des légumes et des fruits, París.

Powell, J. G. F. 2007: «A New Text of the Appendix Probi», CQ 57, pp. 687-700.

Quirk, R. J. 2005: «The Appendix Probi as a Compendium of Popular Latin: Description and Bibliography», $C W$ 98, pp. 397-409.

Reeve, M. D. 2007: «The Editing of Pliny's Natural History», RHT n. s. 2, pp. 107-179.

Rodgers, R. H. 2010: L. Iuni Moderati Columellae res rustica. Incerti auctoris liber de arboribus, Oxford.

Stadler, H. 1910: «Gargilius 1», en RE Pauly-Wissowa VII, Stuttgart, cols. 760-762.

Stotz, P. 1996: Handbuch zur lateinischen Sprache des Mittelalters. Band 3. Lautlehre, Múnich.

Trinquier, J. 2012: «Serpents buveurs d'eau, serpents oenophiles et serpents sanguinaires: les serpents et leurs boissons dans les sources antiques», Anthropozoologica 47, pp. 177-221.

Fecha de recepción de la primera versión del artículo: 31/01/2019

Fecha de aceptación: 28/02/2019

Fecha de recepción de la versión definitiva: 02/04/2019 